\title{
SECURING INFORMATION SYSTEMS A NEW APPROACH
}

\author{
William R. Simpson \\ Institute for Defense Analyses, 4850 Mark Center Dr., Alexandria, \\ Virginia 22311
}

\begin{abstract}
Increasing threat intrusions to enterprise computing systems have led to a formulation of guarded enterprise systems. The approach was to put in place steel gates and prevent hostile entities from entering the enterprise domain. The current complexity level has made the fortress approach to security implemented throughout the defence, banking, and other high trust industries unworkable. The alternative security approach presented in this paper is the result of a concentrated fourteen year program of pilots and research. Its distributed approach has no need for passwords or accounts and derives from a set of tenets that form the basic security model requirements. At each step in the process it determines identities and claims for access and privileges. These techniques are resilient, secure, extensible, and scalable. They are currently being implemented for a major enterprise, and are a candidate for other enterprise security approaches. This paper discusses the Enterprise Level Security architecture, a webbased security architecture designed to select and incorporate technology into a cohesive set of policies and rules for an enterprise information system. The paper discusses the history, theoretical underpinnings, implementation decisions, current status, and future plans for expansion of capabilities and scale.
\end{abstract}

\section{KEYWORDS}

Access control, attributes, authentication, claims, cryptography, digital signatures, enterprise, high assurance, identity management systems, public key infrastructure

\section{INTRODUCTION}

A set of problems exist where continued attempts at eliminating the symptom that results from design complexity has been unsuccessful at achieving the goal of eliminating the symptom.

\subsection{Case Study: Boat Design}

The original builders of boats had a simple design philosophy. Separate the water from the interior of the boat. In the beginning, boats were watertight by virtue of their being hewn from a single tree trunk, or when constructed from more than one piece of wood, joints were sealed with pitch or other sealants. That they leaked was not a big concern. The boats were not used in prolonged activity and did not get far from the shoreline. But as they were developed to move further offshore, some techniques were developed to prevent leaks (shiplap construction, for example) - they still leaked - just enough that it continually had to be dealt with. This limited their effectiveness and time before they needed to be brought ashore. As they got more complex, the leaks were of greater concern. Additional techniques were developed (tongue and grove construction, pitch and other sealants) - they still leaked. Sealants got better, but boats got more

Natarajan Meghanathan et al. (Eds) : ACSIT, SIPM, FCST, CoNeCo, CMIT - 2017 
complex as hatches for cargo, weapons, and steerable rudders mounted through the hull were added. Boats still leaked, not much, but just enough that it limited the range or speed, or time in the water without maintenance. Of course that wasn't going be tolerated! They doubled down (special woods, special formulated sealants, and special paints). They still leaked. A set of boat builders examined the history and came to an epiphany - boats leak. Like all epiphanies, it allows us to re-examine how we handle things. The design was modified so that the inside of boats could accommodate leakage. Drains and channels were added to funnel water to an area, the bilge, where the water could be dealt with, manually at first, and then with automated pumps. A well-sealed boat was still required because a high leakage rate could overwhelm bilge capacity.

\subsection{Case Study: Today}

We find ourselves at a crossroads where the computing systems we have come to rely on, are increasingly vulnerable to attack. Losses have occurred not only at commercial entities such as Target [1], Walmart [2], but at places that should be able to protect our information such as the IRS and FBI [3], OPM [4], etc. Who hasn't been hacked? The answer is surprising and it amounts to nobody. Nobody who is anybody and has something to lose, and somebody who is a nobody with limited presence and little or no assets online. How did we get in this mess?

\subsection{Case Study: Hindsight}

The Advanced Research Projects Agency Network (ARPAnet) was one of the world's first operational packet switching networks, the first network to implement TCP/IP, and the progenitor of what was to become the global Internet. The network was initially funded by the Advanced Research Projects Agency (ARPA, later the Defense Advanced Research Projects Agency (DARPA)) within the U.S. Department of Defense for use by its projects at universities and research laboratories in the United States. The packet switching of the ARPAnet, together with Transmission Control Protocol/Internet Protocol (TCP/IP), formed the backbone of how the Internet works. The packet switching was based on the concepts and designs of engineer Paul Baran, British scientist Donald Davies [5, 6], and Lawrence Roberts of Massachusetts Institute of Technology Lincoln Laboratory [7]. The TCP/IP set of communication protocols was developed for ARPAnet by computer scientists Robert Kahn and Vinton Cerf." [8]

The original ARPAnet connected four computers (1971). They were located in the respective computer research labs of the University of California, Los Angeles (UCLA) (Honeywell DDP 516 computer), Stanford Research Institute (SDS-940 computer), the University of California, Santa Barbara (IBM 360/75), and the University of Utah (DEC PDP-10). As the network expanded, different models of computers were connected, which created compatibility problems. The solution rested in a better set of protocols, TCP/IP, designed in 1982. Under ARPAnet, several major innovations occurred: email (or electronic mail) - the ability to send simple messages to another person across the network (1971); telnet-a remote connection service for controlling a computer (1972); and file transfer protocol (FTP)-allows information to be sent from one computer to another in bulk (1973).

As non-military uses for the network increased, more and more people had access, and it became no longer safe for military purposes. As a result, MILnet, a military-only network, was started in 1983. Internet Protocol software was soon being placed on every type of computer, and universities and research groups also began using in-house networks known as Local Area Networks or LANs. These in-house networks then started using Internet Protocol software so that one LAN could connect to another.

"In 1986, one LAN branched out to form a new competing network, the National Science Foundation Network (NSFnet). NSFnet first linked together the five national supercomputer centers and then every major university, replacing the slower ARPAnet, 
which was finally shut down in 1990. NSFnet formed the backbone of what we now call the Internet".

-A Brief History of Network Computing [9]

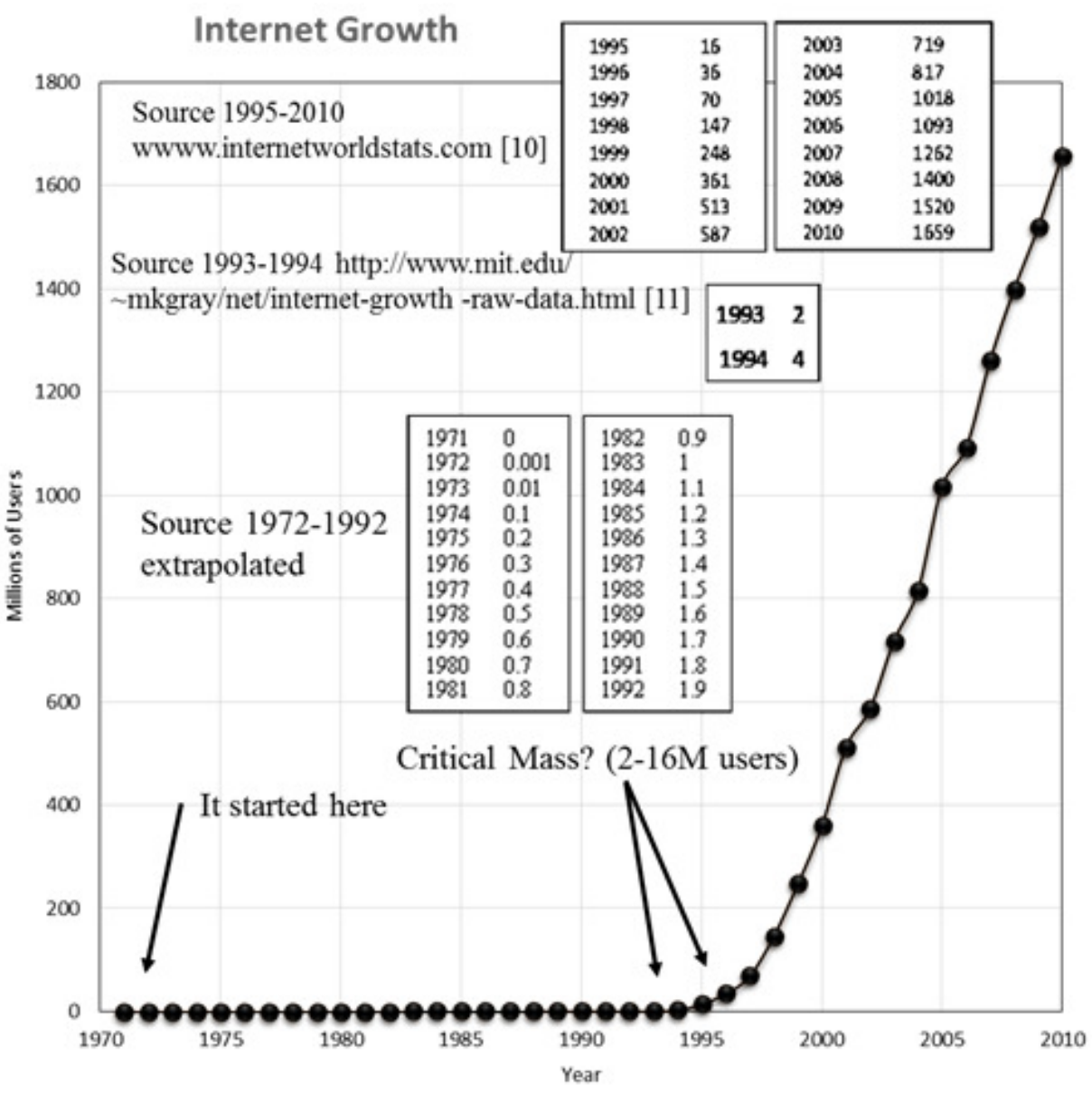

Figure 1. Internet Growth

In the beginning there were no threats only a desire to make computers communicate. This changed quickly. Intruders penetrated unprotected systems. The only thing that grew faster than the internet was the complexity of computing and the internet. Threats were few at first and targeted approaches were used to mitigate them. This worked for a while. Quickly however, more generic methods were needed.

\subsection{Case Study: The Fortress Approach}

The advent of the firewall is the beginning of the fortress approach.

"Acting as a barrier between a trusted network and other untrusted networks -- such as the Internet -- or less-trusted networks -- such as a retail merchant's network outside of a cardholder data environment -- a firewall controls access to the resources of a network through a positive control model. This means that the only traffic allowed onto the network defined in the firewall policy is; all other traffic is denied".

- Firewall [12] 
In the fortress approach a gateway is established that is hardened against all who would enter but were not authorized. This gateway is sometimes called the De-Militarized Zone (DMZ). While the DMZ began with the firewall it certainly didn't end there. Initially somewhat successful, the firewall quickly became ineffective against a number of attackers and the threat still penetrated.

The designers of the DMZ supplemented the DMZ with additional hardware "appliances". These were needed in hardware because they must process everything that comes to the enterprise and needed to operate at "line speeds" so as not to reduce the quality of service provided by the enterprise. The appliances may cost more than $\$ 1$ million each. They added packet inspection to the firewall - the threat still penetrated. Additional techniques (and appliances) were developed (Application aware filtering, white/black and grey listings, signature analysers) - the threat still penetrated. Of course that wasn't going be tolerated! They doubled down (Host based analysers, Intrusion detection, Intrusion prevention) - the threat still penetrated.

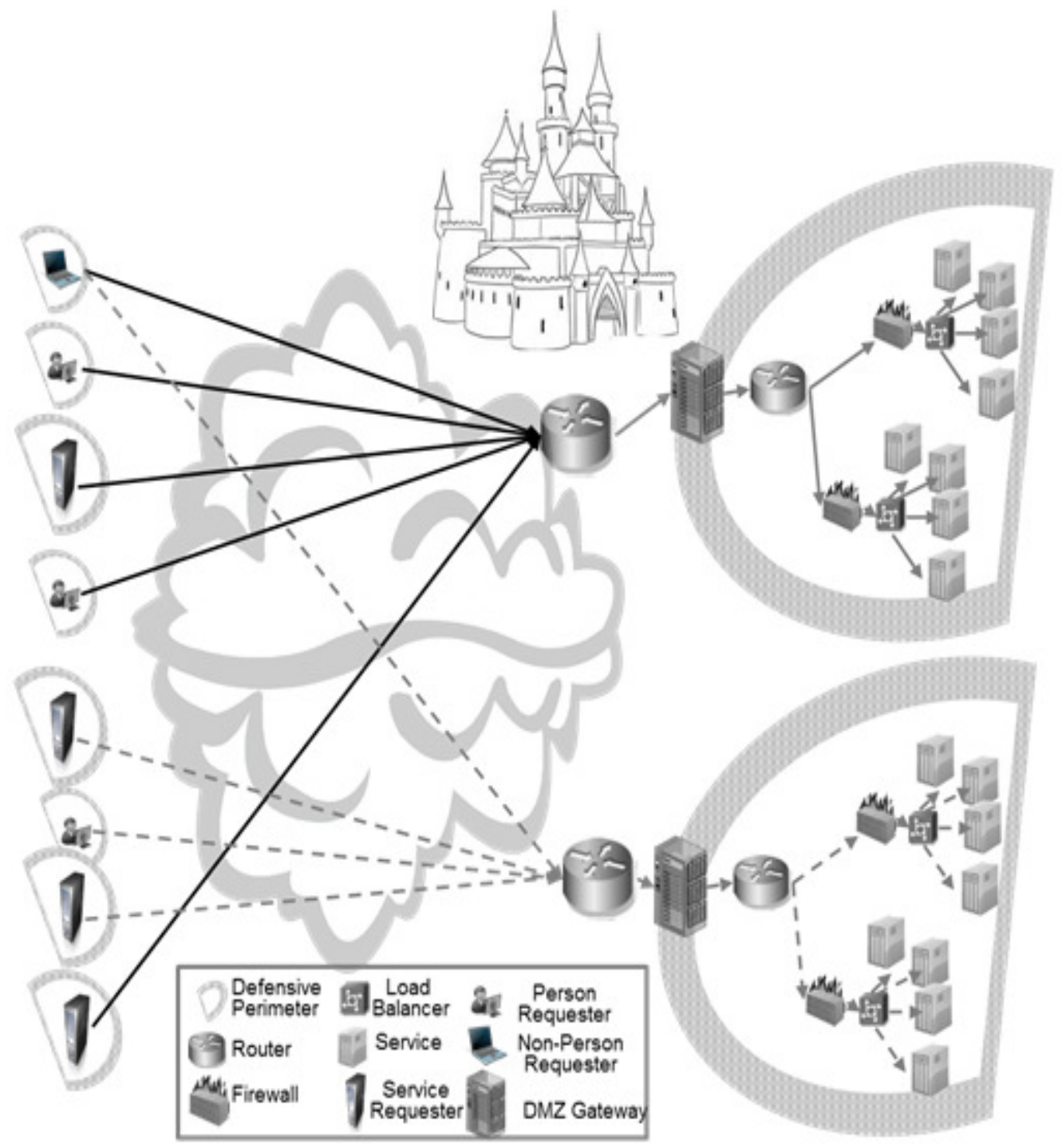

Figure 2. The Fortress Approach (DMZ) 


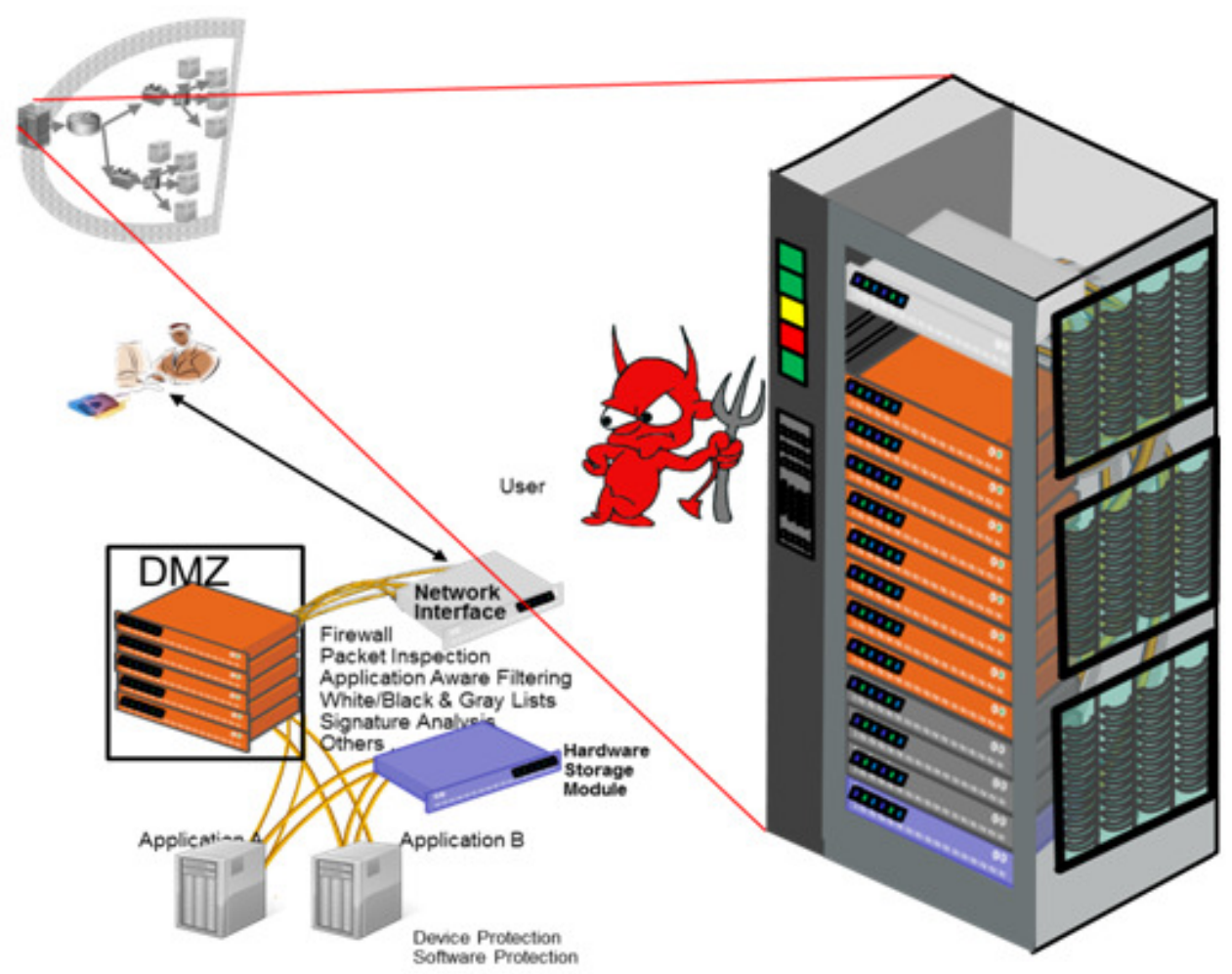

Figure 3. The "Beefed-up" DMZ

Does this sound familiar? It is time for an epiphany -threats penetrate systems. Like all epiphanies, it allows us to re-examine how we handle things.

\section{RE-EXAMINATION OF THE SECURITY APPROACH}

The complexity of modern systems has grown almost without bounds. Complexity makes the system only partially testable. Defence requires preventing everything. Offense requires finding one exploit. Threats cannot be eliminated, only mitigated. Adding complexity in defences exacerbates the problem. Complexity continues and will continue to grow!

The threat is part of the environment.

- Go back to security principles.

- Remove common sources of vulnerabilities (passwords, accounts, escalation of privilege ...).

- Replace passwords with credentials as a basis for trust. Verify and validate all credentials.

- Trust as little as possible. Minimize threat surfaces. Minimize the value of targets (distribute the value among targets).

- Communicate in confidentiality.

- Communicate endpoint to endpoint (no intermediates). Verify that what you received was what was sent (integrity).

- Know with whom you are dealing (no actions on behalf of).

- Know when you have been compromised.

- Monitor and record. Be resilient. ... 
Some basic assumptions have been made at the outset for the security model as derived from the re-examination above. These are:

- Only interactions based on authorization credentials and two-way, end-to-end authentication are permitted [this leads to strong requirements for enterprise naming and credentials];

- Impersonation is not allowed;

- Least Privilege;

- Confidentiality of all data/content exchanged;

- Verify and validate integrity of all communications;

- Monitoring is conducted on all exchanges;

- Eliminate or mitigate Malware by periodic scans and active measures.

The Enterprise Level Security (ELS) has evolved from a fortress approach, where threats are assumed to be stopped at the front door, to a distributed security system that eliminates or mitigates many of the primary vulnerability points within that system, as shown in Figure 4.

\section{Concentrate on the end points.}
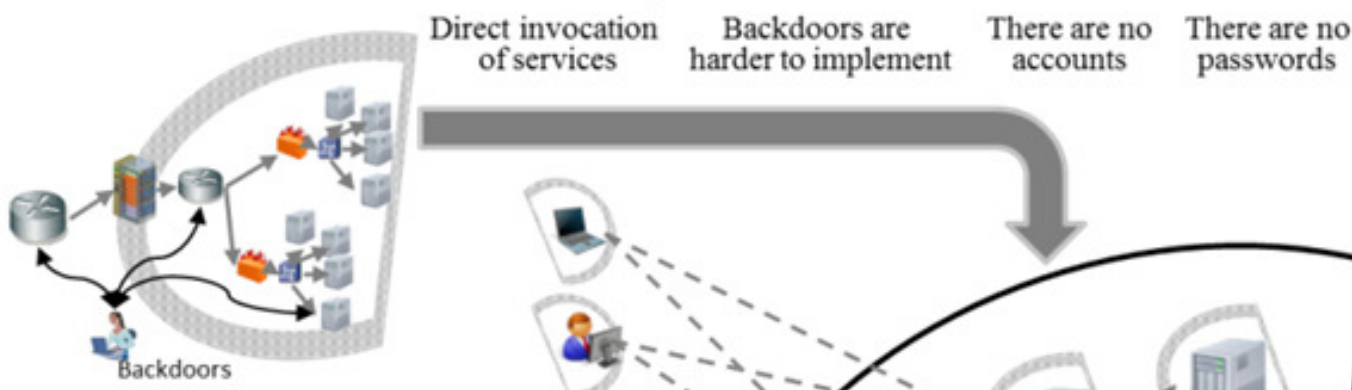

- Reduce the number of high value targets.

- By distributing even if you lose a battle the losses are confined to a small subset of the whole.

- Emphasize backup and recovery.

- Eliminate data warehouses.

- Incorporate federated databases.

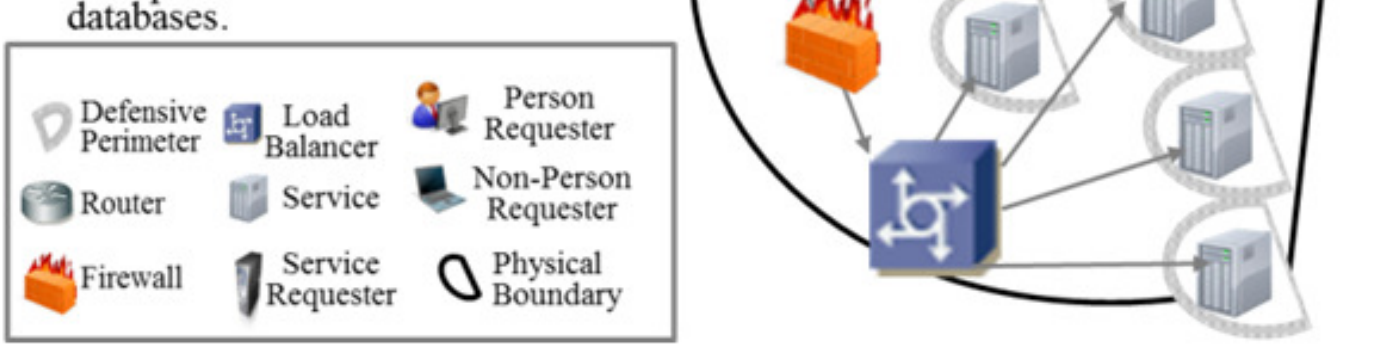

Figure 4. Distributed Security Architecture 


\section{ENTERPRISE LEVEL SECURITY}

ELS is a high-assurance environment. For ELS, we are primarily concerned with five security principles.

- Know the Players - this is done by enforcing bi-lateral end-to-end authentication.

- Maintaining Confidentiality - this entails end-to-end unbroken encryption.

- Separate Access and Privilege from Identity- this is done by an authorization credential

- Maintain Integrity - know that you received exactly what was sent - know that content has not been modified.

- Explicit Accountability - monitor all transactions.

\subsection{Know the Players}

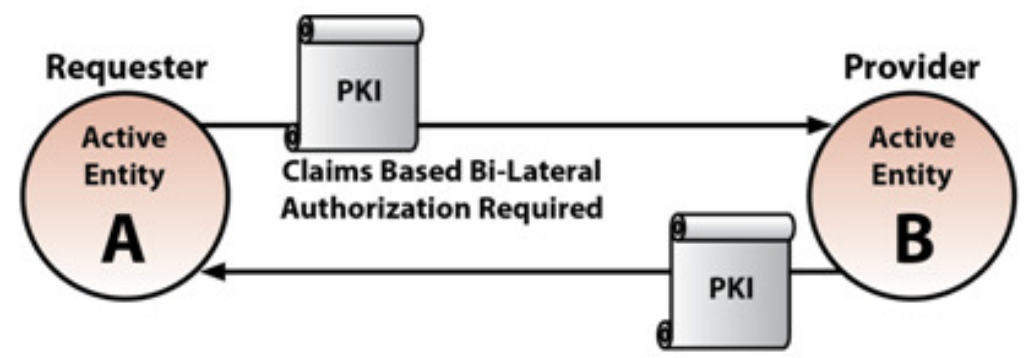

Active Entity may be: User, Web Application, Web Service, Aggregation Service, Exposure Service, Token Server, or any element that can be a requester or provider.

Figure 5. Bi-lateral Authentication

In ELS, the identity certificate is an X.509 PKI certificate [13]. PKI certificates are verified and validated. Ownership is verified by a holder-of-key check.

\subsection{Maintain Confidentiality}

ELS establishes end-to-end TLS [14] encryption (never give away private keys that belong uniquely to the certificate holder). Message authentication codes are enforced (but they are only valid when the encryption remains unbroken to the end point).

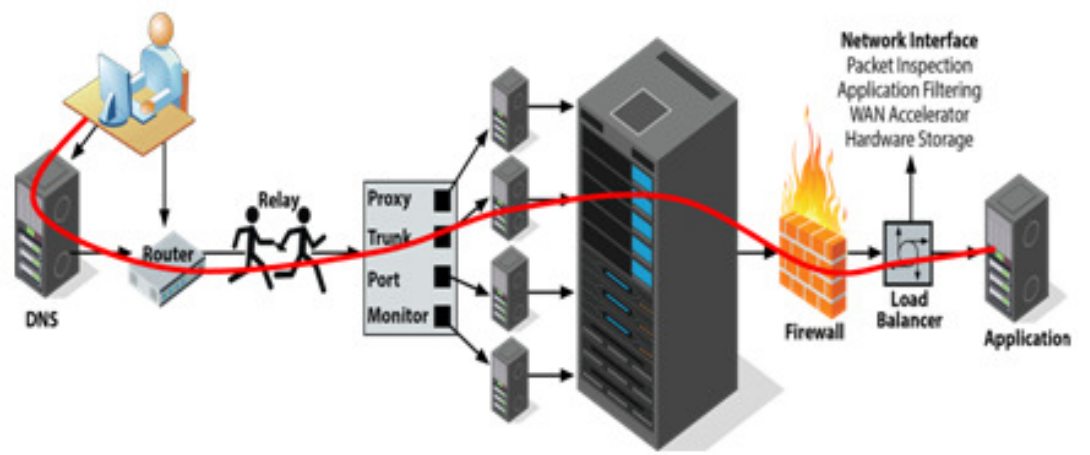

Figure 6. End-to-End Encryption 


\subsection{Separate Access and Privilege from Identity}

In ELS this is accomplished by using the Security Assertion Markup Language (SAML) [15]. SAMLs are signed, and the signatures are verified and validated. The credentials of the signers are verified and validated.

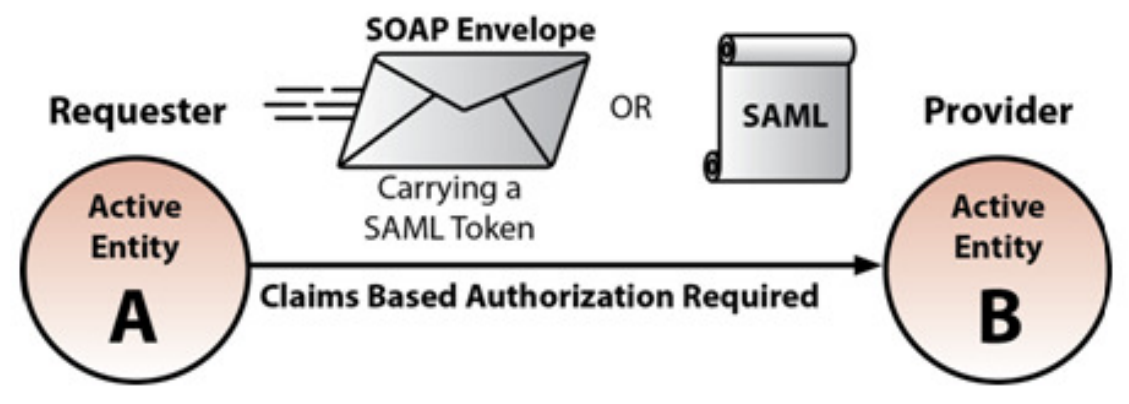

Figure 7. Claims-Based Authorization

\subsection{Maintain Integrity}

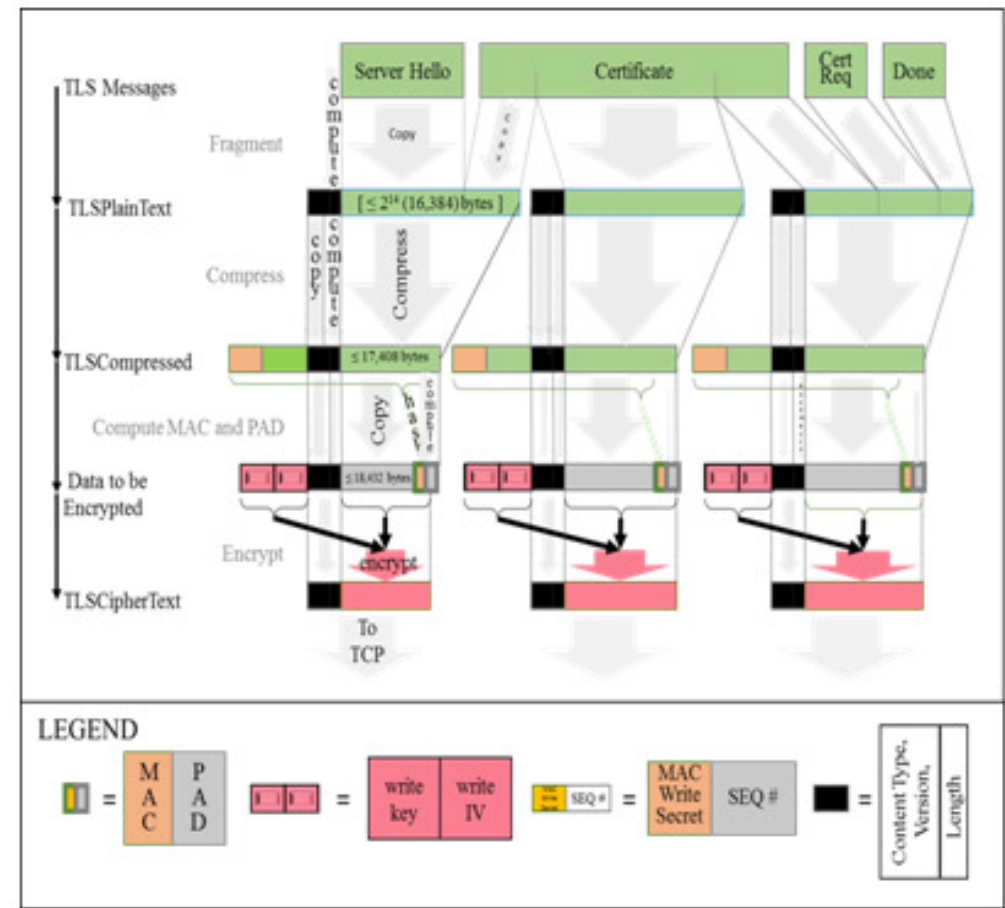

Figure 8. MAC and Other Integrity Measures

In ELS this is implemented by End-to-End TLS encryption with message authentication codes (MAC). Packages (like SAML tokens) are signed, and signatures are verified and validated [16].

\subsection{Require Explicit Accountability}

All active entities must monitor specified activities. For enterprise files a monitor sweep agent reads, translates, cleans, and submits to relational data base for recording log records periodically or on demand. The details of this activity are provided in [17 and 18]. 


\section{InSPECTIONS AND PRotections}

Most appliance functionality is now available as software only. Figure 10 shows the conversion of the particular piece of the inspection chain that applies to a particular server as a pseudo appliance.

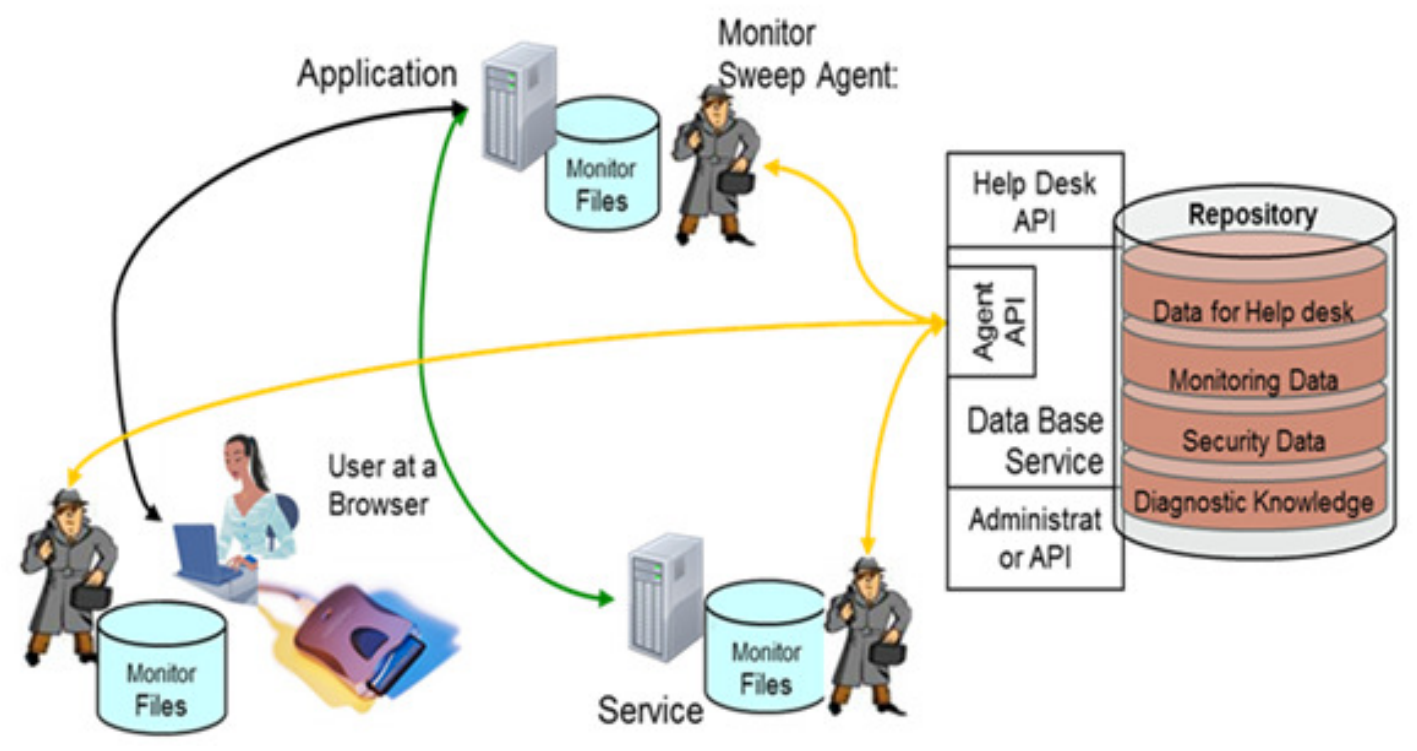

Figure. 9 Accountability through Centralized Monitoring

The packets are decrypted on entry into the pseudo appliance and stay that way with the exception of an offload to an external source (such as a network monitoring appliance where packets are counted and graded, etc. This offloading will be done by a full ELS communication (for security, of course). This sounds pretty similar to the current approach. How does this change anything? The difference is in where the pseudo appliance lives. For the moment let's assume the software appliance lives in the application server. The traffic at the server is considerably less than the front door of the enterprise (obviating the need for lightning speed and hardware only solutions). The inspections can also be tailored (closely inspect some, ignore others) for the specific application.

Of course we cannot send all of the incoming packets to the application server. It would be inefficient and dangerous. Some packets can wreak havoc before they are even processed, so we would want to be sure that the server was the intended recipient and that the server is communicating with a credentialed entity with valid credentials.

Figure 11 has identified an intelligent tagging device that will identify the traffic by observing the first few packets. In the case of official enterprise traffic the first few packets are not encrypted and these involve the exchange of PKI certificates that can be identified and the owners can be compared to a white list. The target will be the destination for traffic. Before identification the packets can be passed through the normal set of inspection appliances - sometimes referred to as the de-militarized zone (DMZ). If no identification is made, the packets will continue through the DMZ. When identified, they can be passed directly to the server or the load balancer in front of the server. 


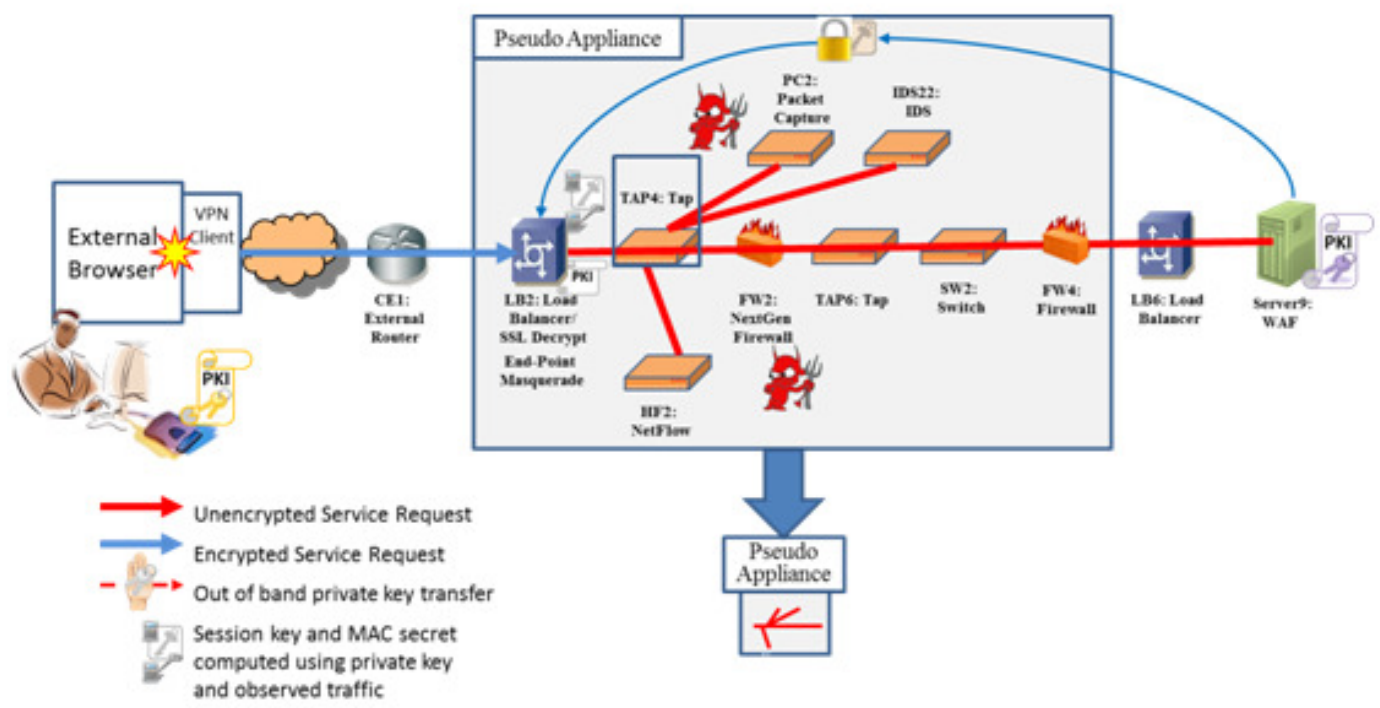

Figure 10. Creation of the Pseudo Appliance

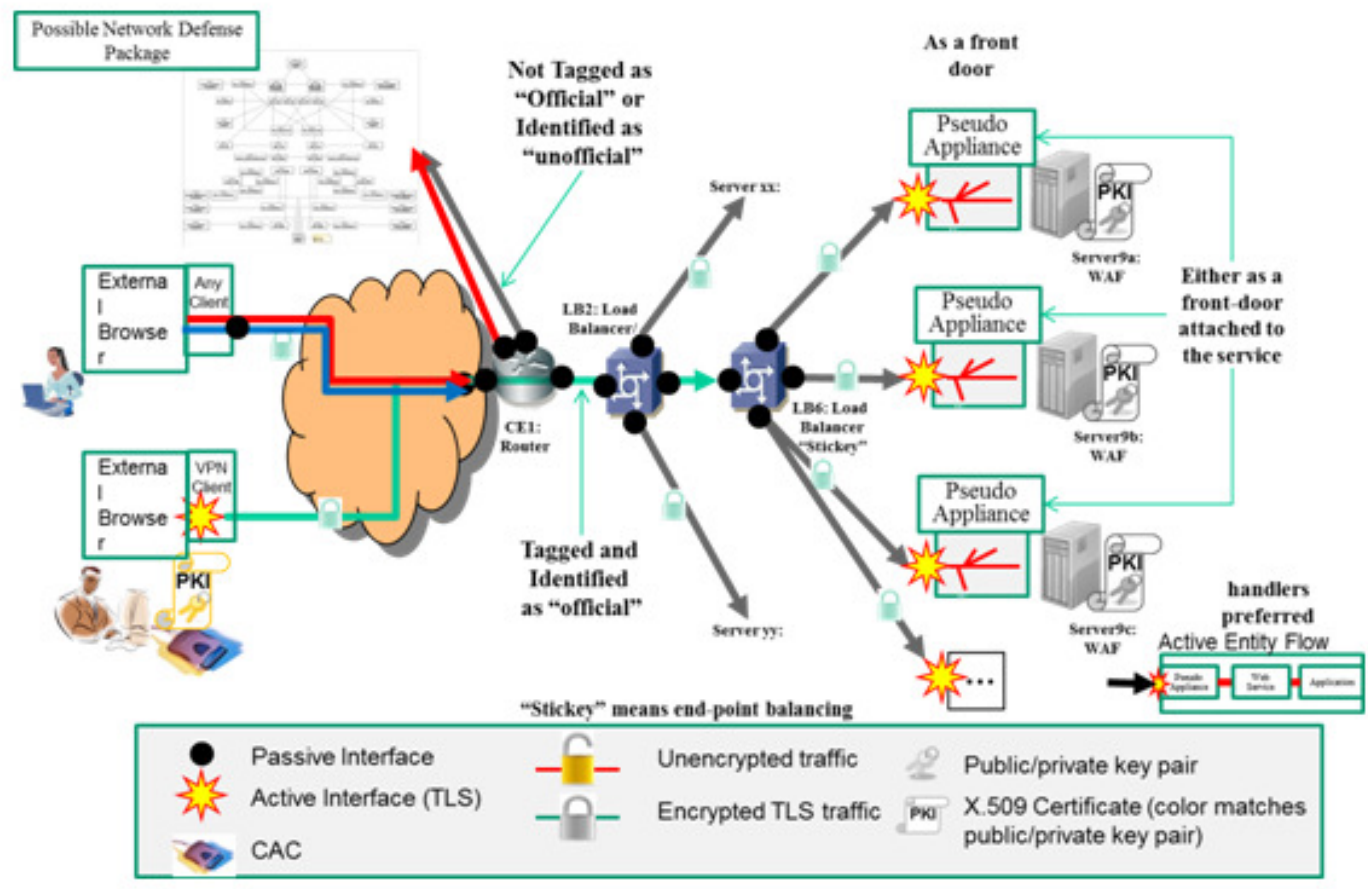

Figure 11. Tagged and Embedded Functionality

The only remaining problem is to reduce the software functionality to handlers in the handler chain as shown in Figure 12. 


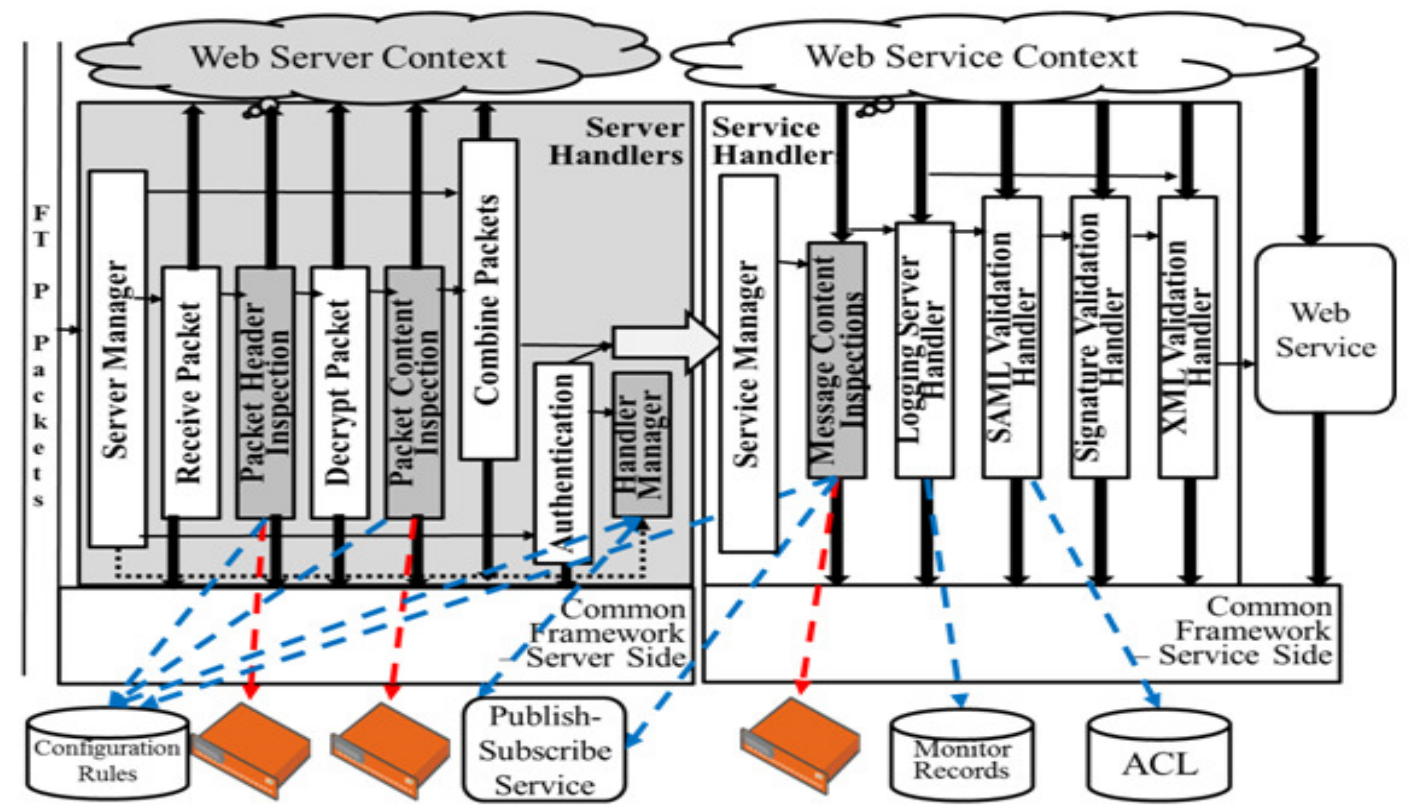

Figure. 12 Server Side Handlers

Note that the handlers are embedded in the server handler chain at the point that the communication is prepared for their use, and that the functionality has been divided along those lines as opposed to the previous functionality such as virus scan, ports and protocols, intrusion detection or blacklist/whitelist, etc. These are distributed to packet header inspection, packet content inspection, and message content inspection. Each of these may perform inspection related to intrusion detection or blacklist blocking, etc. Pilots are being worked on, stay tuned for results. This is the preferred embodiment for enterprise applications. It moves the inspections to the point of the application itself, by inserting handlers within the server and service to do the inspections at the point it makes most sense. The inspections that can be done without decrypting the packets may be done at the front of the web server because they are passive entities. Moving inspections of decrypted traffic inside the server, not only preserves the end-to-end paradigm, but encapsulates the security and allows tailoring for the application itself. The encapsulated security with the application is virtualization ready.

\section{An Evolution}

The ELS is the result of a carefully crafted architecture. The initial research began in 2002 with the original development of design tenets and each of the major components. These include:

- Fully encrypted unbroken end-to-end communications (later defined to be TLS with message authentication codes).

- Bi-lateral PKI authentication for all enterprise entities;

- SAML-based approaches as hardened for vulnerability mitigation for access and privilege;

- Embedded SAML handlers for consistency in application;

- Claims-based access and privilege approach as opposed to attributes and roles;

- Defined federation and delegation processes; 
- Virtualization inspection handles (in process).

A full implementation began in 2015 with a spiral based roll-out leading to pathfinder applications, Cyber range evaluations, and other applications currently in process.

\section{Conclusions}

We have reviewed the basic approaches to the restriction of database access, and the assignment of privilege with databases. The common approach to a web service front end of a Database Management System (DBMS) requires the web service to restrict access and privilege based upon the user context. In doing this it must be provided with full access and privilege to the database, and be trusted to limit user access and privilege. We reviewed the high-assurance security paradigm and the changes that must be made for hardening the security associated with database operations. The suggested approaches build increasing security by adding user-tailored restrictions directly into the database, and they provide the web service fronting the DBMS with the same privilege as the user. At the same time, it restricts SQL queries to a fundamental set that will be enforced by the view developed within the database and not at the web service. A final area, yet to be developed is the application of partial homomorphic techniques that keeps all transactions encrypted.

This research is part of a body of work for high-assurance enterprise computing using web services. Elements of this work include bi-lateral end-to-end authentication using PKI credentials for all person and non-person entities, a separate SAML credential for claims-based authorization, full encryption at the transport layer, and a defined federation process. Many of the elements of this work are described in [19-24]. The entire process has been recently published in [25]

\section{REFERENCES}

[1] Chris Poulin 2014, "What Retailers Need to Learn from the Target Breach to Protect against Similar Attacks”, https://securityintelligence.com/target-breach-protect-against-similar-attacks-retailers/ , accessed on 2/25/1016.

[2]. Kim Zetter 2009, "Big-Box Breach: The Inside Story of Wal-Mart's Hacker Attack", http://www.wired.com/2009/10/walmart-hack/, accessed on 2/25/1016.

[3]. Colleen Weller 2016, "Cyber-Security in 120 Secs: Breaches at FBI and IRS", http://blog.ensilo.com/cyber-security-in-120-secs-breaches-at-fbi-and-irs, accessed on 2/25/1016.

[4]. David Bisson 2015, “The OPM Breach: Timeline of a Hack", http://www.tripwire.com/state-ofsecurity/security-data-protection/cyber-security/the-opm-breach-timeline-of-a-hack/, accessed on $2 / 25 / 1016$.

[5]. Donald W. Davies CBE, FRS, June 7, 1924, Treorchy, UK, May 28, 2000, Australia, http://www.thocp.net/biographies/davies_donald.htm

[6]. INTERNET HALL of FAME PIONEER Donald Davies, http://www.internethalloffame.org/inductees/donald-davies

[7]. "Lawrence Roberts Manages the ARPANET Program." http://www.Living_Internet.com, Retrieved 6 November 2008.

[8]. ARPANET, http://en.wikipedia.org/wiki/ARPANET

[9]. A brief History of Network Computing, http://inventors.about.com/library/weekly/aa091598.htm

[10]. Internet Growth Data 1995-2010, www.internetworldstats.com 
[11]. MIT Internet Growth 1993-1994, http://www.mit.edu/ mkgray/net/internet-growth-raw-data.html, Internet Growth Data 1971-1992, Extrapolated by Author.

[12]. Essential Guide, "Enterprise firewall protection: Where it stands, where it's headed", http://searchsecurity.techtarget.com/definition/firewall, accessed on 2/25/1016.

[13]. X.509 Standards

a. DoDI 8520.2, Public Key Infrastructure (PKI) and Public Key (PK) Enabling, 24 May 2011

b. JTF-GNO CTO 06-02, Tasks for Phase I of PKI Implementation, 17 January 2006

c. X.509 Certificate Policy for the United States Department of Defense, Version 9.0, 9 February 2005

d. FPKI-Prof Federal PKI X.509 Certificate and CRL Extensions Profile, Version 6, 12 October 2005

e. RFC Internet X.509 Public Key Infrastructure: Certification Path Building, 2005

f. Public Key Cryptography Standard, PKCS \#1 v2.2: RSA Cryptography Standard, RSA Laboratories, October 27, 2012

g. PKCS\#12 format PKCS \#12 v1.0: Personal Information Exchange Syntax Standard, RSA Laboratories, June 1999 http://www.rsa.com/rsalabs/node.asp?id=2138 PKCS 12 Technical Corrigendum 1, RSA laboratories, February 2000

[14]. TLS family Internet Engineering Task Force (IETF) Standards

In draft for reference only:

h. TLS Renegotiation Support Extension to HTTP/2, 2015-03-24

i. Terminology related to TLS and DTLS, 2015-03-26

j. X.509v3 TLS Feature Extension, 2015-04-06

k. TLS over HTTP, 2015-03-09

1. A TLS ClientHello padding extension, 2015-02-17

m. Elliptic Curve Cryptography (ECC) Cipher Suites for Transport Layer Security (TLS) Versions 1.2 and Earlier, 2015-03-09

n. Transport Layer Security (TLS) Session Hash and Extended Master Secret Extension, 201504-16

o. The Transport Layer Security (TLS) Protocol Version 1.3, 2015-03-09

p. Standards:

q. RFC 2830 Lightweight Directory Access Protocol (v3): Extension for Transport Layer Security, 2000-05

r. RFC 3749 Transport Layer Security Protocol Compression Methods, 2004-05

s. RFC 4279 Pre-Shared Key Ciphersuites for Transport Layer Security (TLS), 2005-12

t. RFC 5246 The Transport Layer Security (TLS) Protocol Version 1.2, 2008-08

u. RFC 5289 TLS Elliptic Curve Cipher Suites with SHA-256/384 and AES Galois Counter Mode (GCM), 2008-08

v. RFC 5929 Channel Bindings for TLS, 2010-07

w. RFC 6358 Additional Master Secret Inputs for TLS, 2012-01

x. RFC 7251 AES-CCM Elliptic Curve Cryptography (ECC) Cipher Suites for TLS, 2014-06

y. RFC 7301 Transport Layer Security (TLS) Application-Layer Protocol Negotiation Extension, 2014-07

z. RFC 7457 Summarizing Known Attacks on Transport Layer Security (TLS) and Datagram TLS (DTLS), 2015-02

[15]. Organization for the Advancement of Structured Information Standards (OASIS) open set of Standards

a. N. Ragouzis et al., Security Assertion Markup Language (SAML) V2.0 Technical Overview, OASIS Committee Draft, March 2008

b. P. Mishra et al. Conformance Requirements for the OASIS Security Assertion Markup Language (SAML) V2.0. OASIS Standard, March 2005.

c. S. Cantor et al. Assertions and Protocols for the OASIS Security Assertion Markup

Language (SAML) V2.0, OASIS Standard, March 2005 
[16]. William List and Rob Melville, IFIP Working Group 11.5, Integrity In Information, Computers and Security, Volume 13, Issue 4, pp. 295-301, Elsevier, doi:10.1016/0167-4048(94)90018-3, 1994.

[17]. William R. Simpson and Coimbatore Chandersekaran, CCCT2010, Volume II, pp. 84-89, "An Agent Based Monitoring System for Web Services," Orlando, FL, April 2011.

[18]. William R. Simpson and Coimbatore Chandersekaran, 1st International Conference on Design, User Experience, and Usability, part of the 14th International Conference on Human-Computer Interaction (HCII 2011), "A Multi-Tiered Approach to Enterprise Support Services," 10 pp. Orlando, FL, July 2011. Also published in: A. Marcus (Ed.): Design, User Experience, and Usability, Pt I, HCII 2011, LNCS 6769, pp. 388-397, 2011.@ Springer-Verlag Berlin Heidelberg 2011.

[19]. William R. Simpson and Coimbatore Chandersekaran, The 3rd International Multi-Conference on Engineering and Technological Innovation: IMETI2010, Volume 2, "Use Case Based Access Control," pp. 297-302, Orlando, FL., July 2010.

[20]. William R. Simpson and Coimbatore Chandersekaran, International Journal of Computer Technology and Application (IJCTA), "An Agent-Based Web-Services Monitoring System," Vol. 2, No. 9, September 2011, pp. 675-685.

[21]. William R. Simpson, Coimbatore Chandersekaran and Ryan Wagner, Lecture Notes in Engineering and Computer Science, Proceedings World Congress on Engineering and Computer Science 2011, Volume I, "High Assurance Challenges for Cloud Computing," pp. 61-66, San Francisco, October 2011.

[22]. Coimbatore Chandersekaran and William R. Simpson, Lecture Notes in Engineering and Computer Science, Proceedings World Congress on Engineering 2012, The 2012 International Conference of Information Security and Internet Engineering, Volume I, "Claims-Based Enterprise-Wide Access Control," pp. 524-529, London, July 2012.

[23]. William R. Simpson and Coimbatore Chandersekaran, Lecture Notes in Engineering and Computer Science, Proceedings World Congress on Engineering 2012, The 2012 International Conference of Information Security and Internet Engineering, Volume I, "Assured Content Delivery in the Enterprise," pp. 555-560, London, July 2012.

[24]. Coimbatore Chandersekaran and William R. Simpson, International Journal of Scientific Computing, Vol. 6, No. 2, "A Uniform Claims-Based Access Control for the Enterprise," December 2012, ISSN: 0973-578X, pp. 1-23.

[25]. William R. Simpson, "Enterprise level security: securing information systems in an uncertain world", Taylor \& Francis (CRC Press), Boca Raton, Florida, @2016, LCCN 2015041818 ; ISBN 978-1-49876445-2, 26 January 2016.

\section{AUTHOR}

Dr. William R. Simpson earned his bachelor of science in aerospace engineering from Virginia Polytechnic Institute and State University, a master of science and a doctor of philosophy in Aeronautical and Astronautical engineering from Ohio State University, and a master of science in administration from George Washington University. He has held academic positions at George Mason University, Old Dominion University, the University of Maryland, and Ohio State University. He has held industry positions at the US Naval Air Test Center, the Center for Naval Analyses, the ARINC Research Corporation, and the Institute for Defense Analyses.

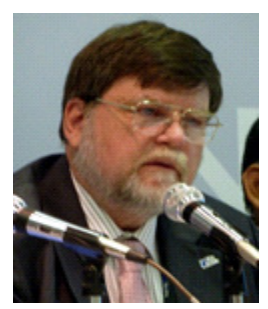

